\title{
Optimising aesthetic outcome after nipple-areola complex-sparing mastectomy and immediate one-stage prosthetic reconstruction: A simple surgical trick to fix nipple-areola complex position
}

\author{
Luca Maione ${ }^{1,2}$, Andrea Lisa ${ }^{1}$, Federico Barbera ${ }^{1}$, Mattia Siliprandi ${ }^{1}$, Valeriano Vinci ${ }^{1}$, \\ Francesco Klinger ${ }^{3}$, Marco Klinger ${ }^{1}$ \\ 1Department of Medical Biotechnology and Translational Medicine BIOMETRA, Plastic Surgery Unit, Humanitas Research \\ Hospital, Reconstructive and Aesthetic Plastic Surgery School, University of Milan, Rozzano (Milan), ${ }^{2}$ Clinica San Carlo, Uo \\ Chirurgia Plastica, Via Ospedale 21, 20037 Paderno Dugnano, Milan, ${ }^{3}$ MultiMedica Holding S.p.A.- Plastic Surgery Unit, \\ Reconstructive and Aesthetic Plastic Surgery School, University of Milan, Sesto San Giovanni (Milan), Italy
}

Address for correspondence: Prof. Marco Klinger, Department of Medical Biotechnology and Translational Medicine BIOMETRA, Plastic Surgery Unit, Humanitas Research Hospital, Reconstructive and Aesthetic Plastic Surgery School, University of Milan, Via Manzoni 56, 20089 Rozzano, Milan, Italy. E-mail: marco.klinger@humanitas.it

\section{ABSTRACT}

Background: Nipple-areola complex (NAC) sparing mastectomy (NSM) is mostly indicated in patients with small-/medium-sized and non-ptotic breasts, while skin-reducing mastectomy is used in patients with medium or large breasts with severe ptosis. NAC location on the reconstructed breast is one of the major factors in determining the final aesthetic result and patients' satisfaction. An optimum result obtained at the end of surgical procedure may be altered and compromised by skin redistribution and consequently NAC depositioning during the post-operative period in patients with medium-sized breasts and a moderate degree of ptosis. Aims: In the present study, we propose a simple surgical trick to fix the NAC in the desired position with a long-lasting result. Methods: We selected 35 patients undergoing NAC sparing mastectomy for breast cancer and immediate one-stage prosthetic reconstruction and we performed a single suture to fix NAC in the desired position before closing the skin envelope. We evaluated NAC complex position stability overtime comparing pre-operative standard photographs with early ( 3 weeks after surgery) and late (1 year after surgery). Results: In all patients, we were able to place the NAC complex on the desired position, and the result was stable at 1 year follow-up. The aesthetic outcome was satisfactory in all patients with no change in the complication rate. Conclusions: This simple surgical trick has been shown to be safe and effective in optimising the aesthetic outcome in a patient undergoing NAC sparing mastectomy and immediate one-stage prosthetic reconstruction.

\begin{tabular}{|l|l|}
\hline \multicolumn{2}{|c|}{ Access this article online } \\
\hline Quick Response Code: & Website: \\
\hline & www.ijps.org \\
\cline { 2 - 2 } & Dol: \\
\hline
\end{tabular}

This is an open access article distributed under the terms of the Creative Commons Attribution-NonCommercial-ShareAlike 3.0 License, which allows others to remix, tweak, and build upon the work non-commercially, as long as the author is credited and the new creations are licensed under the identical terms.

For reprints contact: reprints@medknow.com

How to cite this article: Maione L, Lisa A, Barbera F, Siliprandi M, Vinci V, Klinger F, et al. Optimising aesthetic outcome after nippleareola complex-sparing mastectomy and immediate one-stage prosthetic reconstruction: A simple surgical trick to fix nipple-areola complex position. Indian J Plast Surg 2017;50:64-7. 
Level IV: evidence obtained from multiple time series with or without the intervention, such as case studies. Dramatic results in uncontrolled trials might also be regarded as this type of evidence.

\section{KEY WORDS}

Breast reconstructive surgery; Nipple-areola complex position; nipple-areola complex sparing mastectomy; prosthetic breast reconstruction

\section{INTRODUCTION}

ipple-areola complex (NAC) preservation in female patient undergoing mastectomy for breast cancer may improve patient satisfaction with better cosmetic results. ${ }^{[1-4]}$ This technique was initially described in 2001 as a modification of skin sparing mastectomy, and nowadays, it is performed both as prophylactic or therapeutic surgical procedure. Many studies have revealed the oncological safety of this technique, particularly when the tumor mass is far from NAC $(4-5 \mathrm{~cm}){ }^{[1]}$ NAC preservation has been demonstrated to be related to a better body image perception if compared to skin reducing mastectomy (SRM), thus resulting in decreased psychological discomfort. ${ }^{[2]}$

NAC-sparing mastectomy (NSM) is mostly indicated in patients with small-/medium-sized and non-ptotic breasts, while SRM is usually the best option in patients with medium- or large-sized breasts with severe ptosis, planning surgical incisions as similarly performed during mastopexies or reduction mammaplasties. In both cases, an immediate prosthetic reconstruction can be performed, creating a complete submuscular pocket to cover the breast implant.

The real "no men's land" is represented by patients with medium-sized breasts with a moderate degree of ptosis. In those situations, neither NSM nor SRM are often associated with optimal cosmetic results. ${ }^{[5]}$

NAC location on the reconstructed breast surface is a major factor in determining the final aesthetic outcome and patients' satisfaction. Thus, NAC placement on breast maximum projection point, maintaining the symmetry with the contralateral breast (and its NAC position), has a crucial role in determining the aesthetic outcome. ${ }^{[3]}$

In the present study, we report our personal surgical technique on 35 consecutive patients to maintain NAC position after immediate one-stage breast reconstruction with implants after NAC-sparing mastectomy.
We selected 35 consecutive patients undergoing NSM for breast cancer treatment and immediate one-stage prosthetic reconstruction. The average age was $46 \pm 12$ years (range $30-65$ ) with a minimum 1 year follow-up. After informed consent was obtained, standard pre-operative photographs were taken as follows to evaluate NAC complex position [Figures 1 and 2].

- Lateral view (right and left)

- Oblique view (right and left)

- Anterior-posterior view.

For each patient, we took a pre-operative photograph, an early post-operative photograph (3-4 weeks) after surgery and a late post-operative photograph (1 year after surgery) to assess the stability of NAC position overtime.

After NAC sparing mastectomy was performed, we elevated the pectoralis major muscle; we harvested the recti muscles fascia and the serratus anterior muscle creating a complete submuscular pocket. After accurate haemostasis was performed, we placed two drainages (draining the submuscular pocket and the subcutaneous space, respectively). We finally choose and placed the definitive breast implant and we sutured the muscular layer.

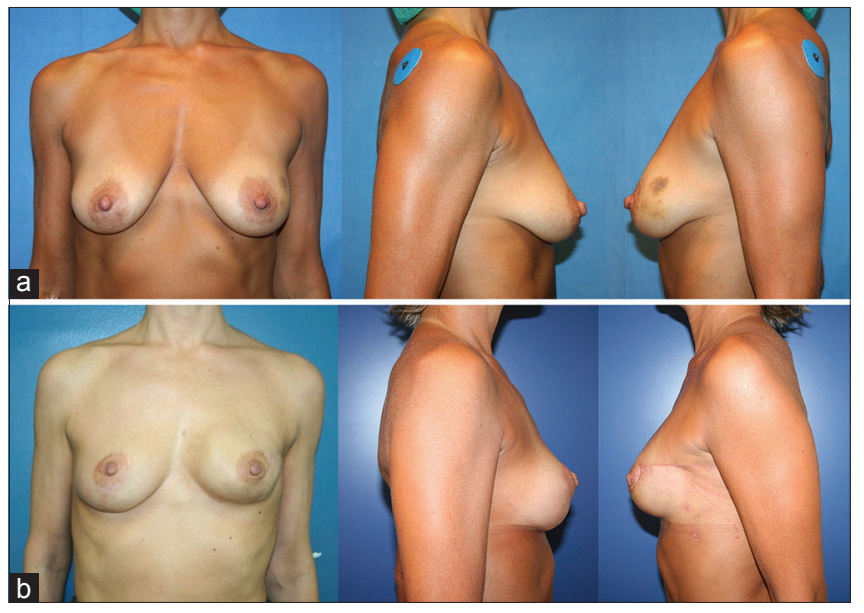

Figure 1: (a) Pre-operative standard photograph (anterior/posterior view - lateral view). (b) Post-operative late standard photograph (anterior/ posterior view - lateral view) 
Afterwards, we placed a suture stitch with the absorbable material (polyglactin) pinching the retro-areolar tissue and fixing the NAC by hooking it at the pectoralis major fascia in the desired position [Figure 3]. Subsequently, a classic intradermal suture was performed. Steri-strips were then placed over the suture and a compression garment was applied.

The reconstructive outcome was evaluated comparing pre-operative and post-operative photographs, and the results were assessed independently by five plastic surgeons with expertise in breast reconstructive surgery considering NAC position and symmetry. Patients' short-term and long-term satisfaction were evaluated according to a 10-point Likert scale, and the questionnaires were administered to the patients every 6 months on the $1^{\text {st }}$ year after surgery.

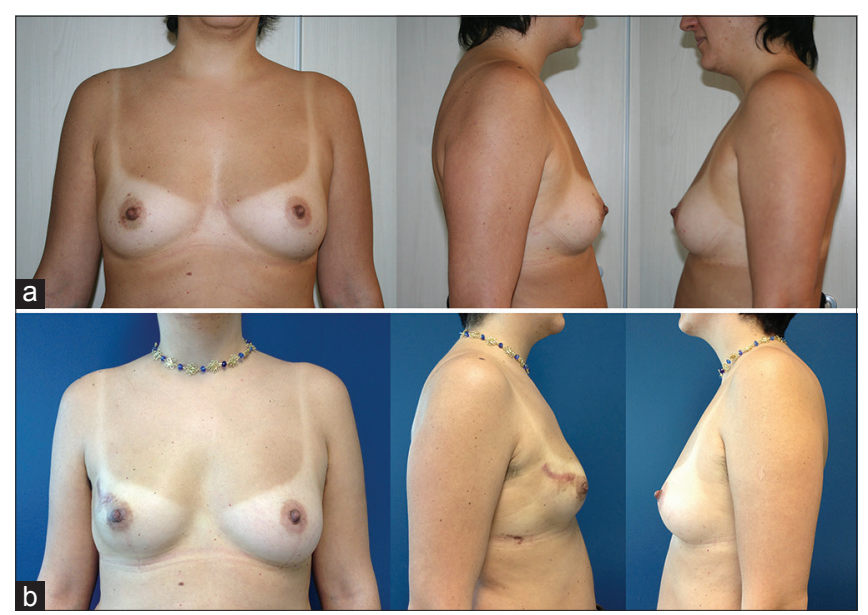

Figure 2: (a) Pre-operative standard photograph (anterior/posterior view - lateral view). (b) Post-operative late standard photograph (anterior/ posterior view - lateral view)

\section{RESULTS}

In all patients, we were able to place the NAC in the desired position on the maximum projection point of the reconstructed breast. This result was stable at 1 year follow-up in all patients despite skin redistribution overtime preventing NAC depositioning [Figures 1 and 2]. The aesthetic outcome was satisfactory in all patients providing a stable symmetry with the contralateral breast with high short-term and long-term patients' satisfaction. We did not observe any increase in the complication rate such as infection, seromas and haematoma, and we had no partial or total NAC necrosis.

\section{DISCUSSION}

The final aesthetic outcome in patients undergoing NAC sparing mastectomy and immediate one-stage prosthetic reconstruction is influenced by a great deal of factors such as the selection of the most appropriate implant, accurate preservation of the NAC vascularization and a proper symmetrisation mammoplasty. Furthermore, NAC position is a leading factor determining the final reconstructive outcome and patient satisfaction, and its stability overtime is a crucial issue that needs to be addressed during the reconstruction. In our experience, patients with small-sized non-ptotic breasts usually have an optimal pairing between muscular and cutaneous layers with a correct NAC positioning at the maximum projection point on the reconstructed breast surface. Conversely, in patients with medium-sized breast and medium-degree ptosis undergoing NSM, we observed skin wrinkling caused by cutaneous

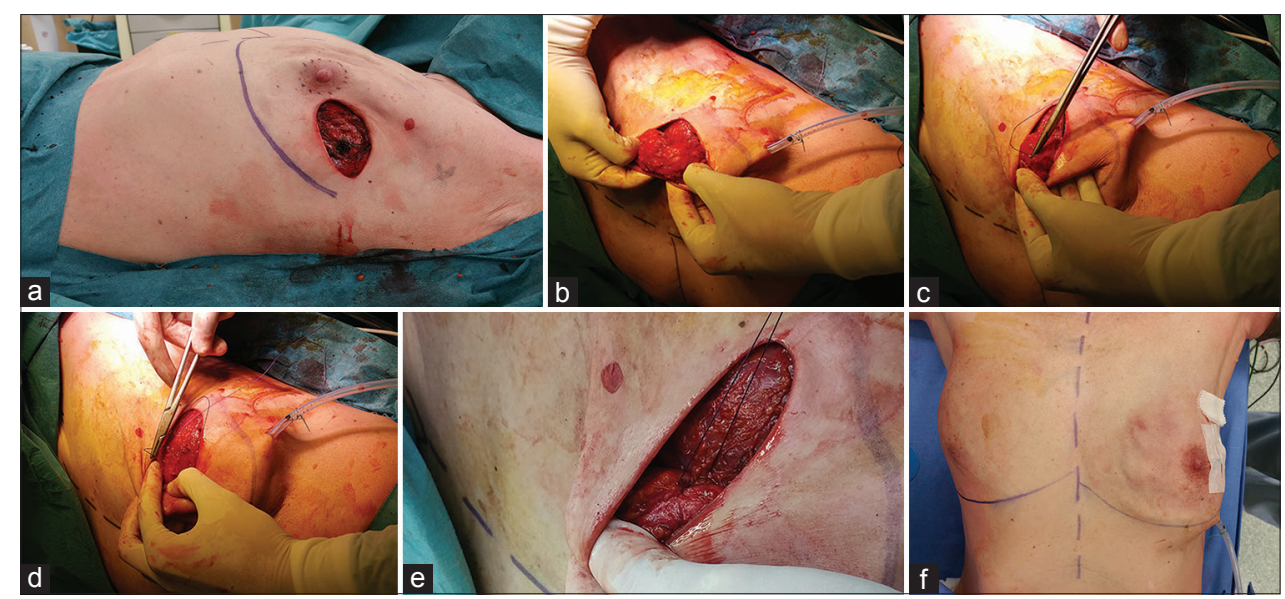

Figure 3: (a) Nipple-areola complex - sparing mastectomy surgical incision. The implant has been placed under a complete submuscular pocket. (b) The posterior aspect of the nipple-areola complex is shown. (c) Suture is taken on the posterior aspect of the nipple-areola complex. (d) Suture is taken on the pectoralis major muscle fascia. (e) Suture is running from the posterior aspect of nipple-areola complex to the pectoralis major fascia preventing nipple-areola complex depositioning after skin redistribution on the post-operative period. (f) The nipple-areola complex is fixed on the maximum projection point on the breast surface 
excess with subsequent imperfect pairing between muscular and subcutaneous layers. During the early post-operative period, spontaneous skin redistribution over the reconstructed breast surface often corrects cutaneous excess thus resulting in a significant NAC depositioning from the ideal maximum projection point of the breast.

Planning a correct surgical incision together with the breast surgeon, choosing the proper permanent implant, careful preservation of NAC vascularisation and accurate contralateral symmetrisation mammoplasty patient's selection are the main factors associated with an optimal reconstructive outcome after NSM. Nevertheless, in medium-sized/moderate ptotic breasts, the final result can be significantly compromised by the previously mentioned skin redistribution and NAC depositioning during the post-operative period. In patients with the cutaneous excess described above, we tried to partially "fix" the NAC position above the reconstructed breast applying steri-strips that pulled the skin in the desired direction during the first 15-20 days after surgery. Results obtained, albeit sometimes discrete, have proved to be often inconstant, not long-lasting, only partially satisfactory and however, not effective and stable in all patients. Thus, we tried to find a technical trick to make NAC position stable overtime. We, therefore, devised an easy manoeuvre allowing us to achieve our target.

In our study, we report a simple and quick technical shrewdness that has been shown to be totally devoid of early and late complications. We did not experience any increase in the rate of prosthesis exposure and reconstruction failure, infection, seromas and partial or total NAC.

\section{CONCLUSION}

Our experience in performing, this simple surgical technique is constantly growing, and patients enroled in long-term follow-up show a great stability of the NAC position overtime. Our post-operative observations are very encouraging, and we state that this technique is safe and effective contributing to an optimal aesthetic outcome with a high satisfaction rate among patients undergoing NSM and immediate breast prosthetic reconstruction.

We, therefore, believe that this simple surgical trick should be performed in all patients to optimise long-term reconstructive outcome.

\section{Financial support and sponsorship}

Nil.

\section{Conflicts of interest}

There are no conflicts of interest.

\section{REFERENCES}

1. Cense HA, Rutgers EJ, Lopes Cardozo M, Van Lanschot JJ. Nipple-sparing mastectomy in breast cancer: A viable option? Eur J Surg Oncol 2001;27:521-6.

2. Didier F, Radice D, Gandini S, Bedolis R, Rotmensz N, Maldifassi A, et al. Does nipple preservation in mastectomy improve satisfaction with cosmetic results, psychological adjustment, body image and sexuality? Breast Cancer Res Treat 2009;118:623-33.

3. Gabka C, Bohmert H. Plastic and Reconstructive Surgery of the Breast. $2^{\text {nd }}$ ed. New York Thieme; 2009.

4. Patani N, Devalia H, Anderson A, Mokbel K. Oncological safety and patient satisfaction with skin-sparing mastectomy and immediate breast reconstruction. Surg Oncol 2008;17:97-105.

5. Rivolin A, Kubatzki F, Marocco F, Martincich L, Renditore S, Maggiorotto F, et al. Nipple-areola complex sparing mastectomy with periareolar pexy for breast cancer patients with moderately ptotic breasts. J Plast Reconstr Aesthet Surg 2012;65:296-303. 